\title{
Composites
}

\section{Enhancement of the electrical and thermal properties of unidirectional carbon fibre/epoxy laminates through the addition of graphene oxide}

\author{
Evangelos C. Senis ${ }^{1, \star}$ (D), Igor O. Golosnoy ${ }^{1}$ (D), Janice M. Dulieu-Barton ${ }^{1}$ (D), and \\ Ole T. Thomsen ${ }^{1}$ (D) \\ ${ }^{1}$ Faculty of Engineering and Physical Sciences, University of Southampton, Southampton, UK
}

Received: 5 December 2018

Accepted: 8 March 2019

Published online:

14 March 2019

(C) The Author(s) 2019

\begin{abstract}
A means of enhancing electrical and thermal conductivities of carbon fibre reinforced polymer (CFRP) composites is investigated for the purpose of reducing damage when electric current and/or heat is introduced into a CFRP structure. The addition of commercially available graphene oxide (GO) nanoflakes dispersed into an epoxy resin is studied; quantities up to $6.3 \mathrm{vol} \%$ are used in a vacuum infusion process with carbon fibre fabric to form CFRP laminates. Measurements of the anisotropic electrical and thermal conductivity of the laminate were conducted on CFRP specimens with and without the GO nano-flakes. It is shown that the electrical conductivity in the through-thickness direction increased markedly, reaching values up to $0.18 \mathrm{~S} / \mathrm{cm}$, when $6.3 \mathrm{vol} \%$ of GO was added into the epoxy, showing a threefold increase compared to the neat CFRP. Similar improvement was also found in the thermal throughthickness conductivity for the same filler content, where the laminate exhibited identical values in both transverse and through-thickness directions. However, the properties transverse to the fibres were not greatly affected by the GO addition. To assess the effect of the GO on the mechanical properties, interlaminar shear strength tests were conducted that showed that the addition of the GO significantly enhanced the through-thickness shear strength.
\end{abstract}

\section{Introduction}

Polymer composite materials reinforced with continuous carbon fibres are being widely used for weight critical structures due to their excellent specific strength and stiffness. However, carbon fibre reinforced polymers (CFRP) exhibit anisotropic mechanical, electrical and thermal properties, which often make their design and application more complex compared to more conventional engineering materials, such as metallic alloys. The increasing demand for CFRP structural solutions in aerospace/ aircraft, automotive and wind turbine blade applications has largely been based on mechanical design

Address correspondence to E-mail: e.senis@soton.ac.uk 
considerations. However, significant new challenges associated with heat dissipation and electric current flow paths have emerged which link directly to operational considerations such as lightning strike protection and anti/de-icing measures [1-3]. The incorporation of a semi-conducting phase (i.e. carbon fibres) into an insulating matrix (i.e. epoxy resin) results in anisotropic thermal, electrical as well as mechanical behaviour in the composite system. The properties parallel to the reinforcement fibres are dominated by the fibre properties, hence the high strength and stiffness, as well as high electrical and thermal conductivities in this direction. On the other hand, transverse to the fibres and in the laminate through-thickness directions, the properties are dominated by the polymer matrix, which exhibits much lower electrical and thermal conductivities than in the direction parallel to the carbon fibre reinforcement [4].

In recent years, several approaches have been suggested to improve the functionality of CFRP laminates using various conductive fillers [2, 5-7]. These include enhancing of the through-thickness electrical and thermal properties as well as the stress transfer between the matrix and carbon fibre. The key objective behind the addition of conducting fillers/ inclusions to the bulk matrix of composite laminates is to interconnect the adjacent laminae with the addition of a secondary conducting phase.

Different methods for improving the conductive performance have been proposed, which show promising results including the incorporation of nano-fillers in the bulk matrix [7-9], grafting on the surface of the carbon fibres by adding nano-inclusions into the sizing $[5,6,10]$, growing nano-inclusions directly on the carbon fibre surface through a chemical vapour deposition (CVD) process [4], electrophoretical deposition of the filler on fabrics [11], and nano-structuring in the interlaminar region $[1,12]$. Although there are differences in the methods listed above, they all share a common characteristic: the use of a conducting filler as a means to enhance the electrical and thermal conductivity of the polymer matrix or the properties of the sizing on carbon fibres [10]. Besides this, parameters such as the interlaminar polymer region and the sizing system have been identified as factors that affect the conduction process either between the laminae or adjacent fibres $[1,10]$.
Carbonaceous inclusions, like, e.g., carbon nanotubes (CNTs) [8, 13-15], carbon black (CB) [1], graphene nano-platelets (GNPs) [7, 12, 16] and carbon nano-fibres (CNFs) [17], as well as metallic nanoparticles or nano-structures, like, e.g., silver nanoparticles (SnPs) and silver nano-wires (SnWs) [7], have been investigated. It has been observed that fillers with high aspect ratios, rod/wire like shapes or platelets/ellipsoids are the most well suited since they can interconnect adjacent fibres and adjacent laminae in the composite system, facilitating both current flow and heat dissipation into the laminate bulk [18].

Graphene oxide (GO) is a 2D material with a similar structure to graphene. It is produced by the oxidation of graphite; hence, the produced sheets are covered with oxygen. Due to its chemical structure, with hydroxyl and epoxide groups on its basal plane and carbonyl and carboxyl groups on the sheet edges, GO is highly compatible with epoxies, unlike graphene, which tends to form agglomerates due to intermolecular van der Waals forces [19-21]. Although GO appears as a promising filler candidate, the oxygen content of GO potentially can affect its electrical and thermal properties, and, therefore, a balance must be established if both good dispersion and improved conductivity properties are required $[22,23]$. So far, the studies utilising GO as reinforcement involved using a laboratory-synthesised filler based on Hummers' method or its variations [24].

To avoid arcing inside the blades, during a lightning strike on a wind turbine blade, the lightning current is split between the down conductor of the lightning protection system (LPS) of the wind turbine and the CFRP structure $[25,26]$. By allowing electric current to flow through the CFRP spar, resistive heating will occur, the extent of which depends on the current density, thus making heat dissipation and current distribution of high importance. Repairs to the internal structure of wind turbine blade are very costly and time-consuming to perform, and lightning strike occurs on numerous occasions over the expected 25-year service life of the wind turbine system. A means of reducing the damage by modifying the material properties is an attractive proposition with improvements not only limited to lightning protection but also to the mechanical response of the structure [27, 28].

The focus of the study described in the present paper is to investigate the influence of GO nano- 
inclusions on the electrical and thermal conductivity in the transverse and through-thickness directions of unidirectional CFRPs when the filler is introduced into the laminate by means of bulk matrix modification. Outside the scope of the paper, but a very important consideration, is the practical implementation of the inclusion of the GO filler materials in epoxy resin at an industrial scale relevant to wind turbine blades. The size of the blade components in the primary load-carrying structure, such as the spar caps and other load-carrying parts, is a key concern. It is essential that the GO filler material is incorporated in a uniform manner in the resin infusion manufacturing process, to deliver a viable approach for lightning strike protection/damage mitigation of the CFRP structure.

\section{Materials and methods}

\section{Materials}

A two-component epoxy system supplied by BASF was used as the matrix material, comprising Baxxores ${ }^{\circledR}$ ER 5300 epoxy resin and Baxxodur ${ }^{\circledR}$ EC 5310 curing agent. The components were mixed by weight at a ratio of $100 / 20$ according to the specifications of the manufacturer. The GO (edge oxidised) was provided by Garmor Inc, USA, consisting of approximately ten graphene layers and a nominal particle size diameter of $500 \mathrm{~nm}, 90 \%$ of the particle sizes below $800 \mathrm{~nm}$, with an oxygen content in the range of $5-10 \%$. A unidirectional (UD) non-crimp carbon fabric used in the manufacturing of wind turbine blade spars, with Zoltek Panex 35 carbon fibres and an areal weight of $882 \mathrm{~g} / \mathrm{m}^{2}$, was used as reinforcement. The composition of the fabric consisted of $852 \mathrm{~g} / \mathrm{m}^{2}$ Panex 35 carbon fibres, $24 \mathrm{~g} / \mathrm{m}^{2}$ of E-glass and $6 \mathrm{~g} / \mathrm{m}^{2}$ of PES, the E-glass and PES were used for stitching purposes.

\section{Manufacturing of GO-reinforced CFRP}

The GO nano-particles were added into the epoxy in pre-specified quantities, as shown in Table 1. The dispersion of GO into the epoxy was realised by means of high-speed planetary mixing, in a dual asymmetric centrifuge configuration, Flacktek Speedmixer ${ }^{\mathrm{TM}} \mathrm{DAC} 150.1 \mathrm{FV}$, for $10 \mathrm{~min}$ at $3000 \mathrm{rpm}$ at ambient temperature. This method introduces high
Table 1 Composition of manufactured GO-reinforced CFRP laminates (matrix volume $V_{\mathrm{m}} \%$ and $V_{\mathrm{GO}} \%$ are estimated based on the volume of GO dispersed into the matrix)

\begin{tabular}{llll}
\hline Sample/GO vol\% in matrix & $V_{\mathrm{f}} \%$ & $V_{\mathrm{m}} \%$ & $V_{\mathrm{GO}} \%$ \\
\hline Neat & 57 & 43 & 0 \\
$0.32 \mathrm{vol} \%$ & 57 & 42.87 & 0.14 \\
$0.63 \mathrm{vol} \%$ & 57 & 42.73 & 0.27 \\
$1.26 \mathrm{vol} \%$ & 57 & 42.46 & 0.54 \\
$2.52 \mathrm{vol} \%$ & 57 & 41.92 & 1.08 \\
$3.78 \mathrm{vol} \%$ & 57 & 41.37 & 1.63 \\
$6.3 \mathrm{vol} \%$ & 57 & 40.29 & 2.71 \\
\hline
\end{tabular}

shear forces into the mixture and has been proven to provide good dispersion of carbonaceous nano-inclusions in epoxy [29-32]. After mixing, the curing agent was added and the mixture was hand stirred for $5 \mathrm{~min}$ followed by degassing at ambient temperature for $10 \mathrm{~min}$. Considering the high areal weight of the reinforcement, the vacuum assisted resin infusion moulding (VARIM) process was used; also, this is the predominant manufacturing route for the manufacturing of wind turbine blades and their structural components [27]. The resin inlet channel was positioned parallel to the fibre direction, Fig. 1. After the infusion process, the laminates were cured for $6 \mathrm{~h}$ at $70{ }^{\circ} \mathrm{C}$, as specified by the manufacturers. The fibre volume fractions given in Table 1 were determined by taking samples from the finished laminates and conducting microscopy, using an Olympus BX-51 optical microscope and Olympus Stream Essentials software, and, in all cases, it was approximately $57 \pm 2 \%$. In addition to the GO filler contents listed in Table 1, trials with higher filler contents of up to $10 \mathrm{vol} \%$ GO were made. The outcome was partially infused laminates, as the resin viscosity increased significantly with the higher volumes of filler, indicating that the mixture was close to its percolation threshold. Thus, the optimum GO filler content for infusion purposes will be below the percolation threshold of that of the GO/epoxy system.

\section{Experimental techniques}

\section{Morphological characterisation}

The morphology of the manufactured samples was investigated by means of scanning electron 
Figure 1 GO-reinforced laminates manufacturing route.

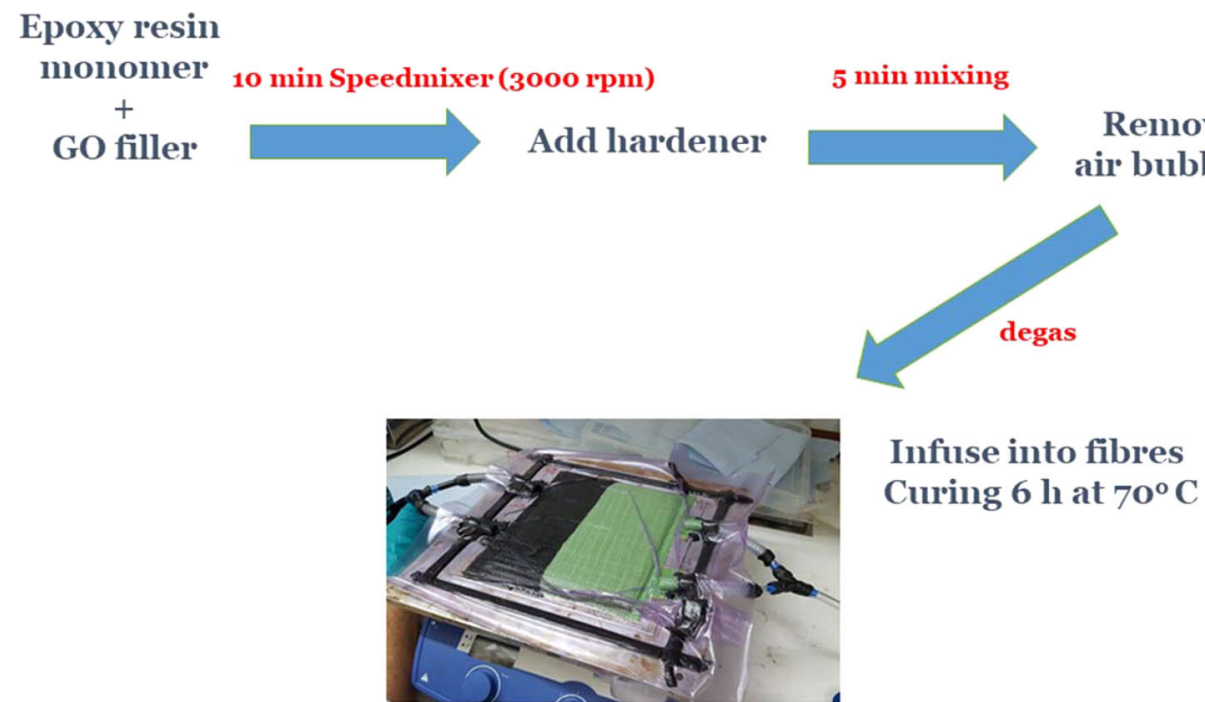

microscopy (SEM), Carl Zeiss EVO 50. Initially, CFRP samples were cut from the manufactured plates using a water-cooled diamond disc wheel cuter before getting sliced, using a diamond saw. Prior to being coated with $\mathrm{Au}$ by sputtering, the samples were submerged in acetone and sonicated for $2 \mathrm{~min}$ to remove any excess dust from the slicing/cutting process.

\section{Electrical conductivity measurements}

The DC resistance was measured with a two-probe set-up with the use of a TTi BS-407 precision milli/ micro-ohmmeter. The applied electric current during the measurements was $5 \mathrm{~mA}$. For the measurements along and transverse to the fibres, rectangular samples were made from the manufactured laminates using a diamond saw (30-180 mm length $\times 40 \mathrm{~mm}$ width $\times 4.2 \mathrm{~mm}$ thickness). The fixture for the electrical conductivity measurements is shown in Fig. 2. The fixture is PTFE (Polytetrafluoroethylene) and includes a screw actuator to control the applied pressure on the electrical contacts to aid reproducibility. To determine the applied pressure level required for repeatable contacts, resistance measurements were obtained as the applied torque was varied and it was shown that above $5 \mathrm{MPa}$ of contact pressure, the resistance exhibited saturation. Hence, the applied pressure was maintained at $5 \mathrm{MPa}$ during the measurements. Measurements in the throughthickness direction were realised using disc-shaped samples (50 mm diameter and $4.2 \mathrm{~mm}$ thickness) with the use of round electrodes featuring a guard

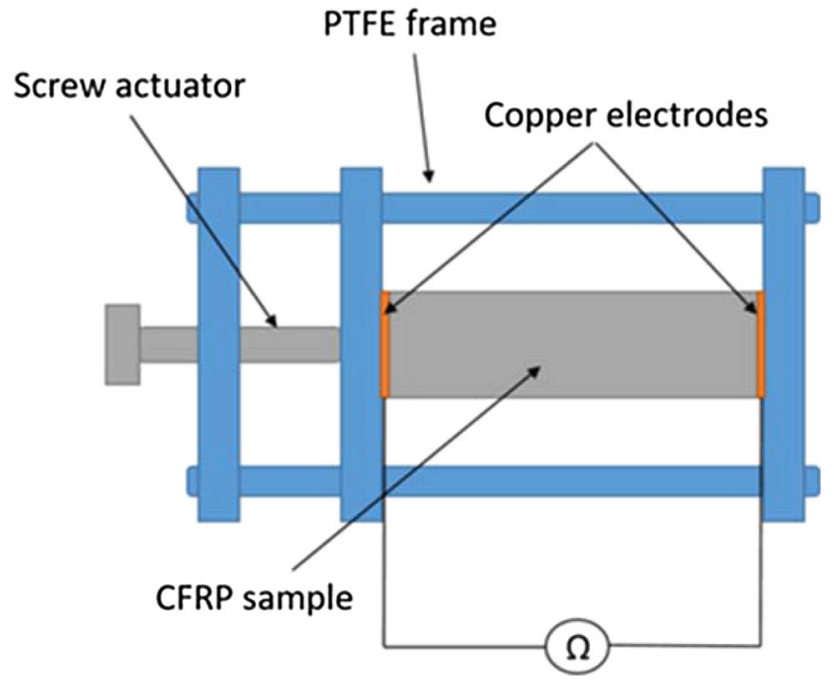

Figure 2 Schematic diagram of the test fixture used for the electrical conductivity measurements.

ring in the voltage-sensing electrode according to ASTM D-257. Due to size and shape of the electrodes, a hydraulic press was utilised to control the required amount of applied pressure. Both electrodes were electrically insulated with PTFE sheets to avoid current leakage to the press.

Prior to the measurements, the sample surfaces that were intended to act as electrodes were initially polished with silicon carbide sandpaper, 600 and 1000 grit, to remove the excess polymer from the surface and expose the carbon fibres to achieve direct electrical contact. To mitigate the influence of surface roughness and promote ohmic contact across the electrode area, an epoxy-based adhesive containing 
silver particles, supplied by RS Components and with a volume electrical conductivity of $10^{3} \mathrm{~S} / \mathrm{cm}$, was employed. Thin layers of the adhesive were applied to the intended electrode surface followed by curing at ambient temperature for $24 \mathrm{~h}$. The current was injected via copper foils, with a thickness of $35 \mu \mathrm{m}$, which were bonded to the test fixture. All the measurements were conducted at a temperature of $25{ }^{\circ} \mathrm{C}$ and $55 \% \mathrm{RH}$. Estimations of the contact resistance were realised by conducting resistance measurements in CFRP samples with constant cross section by varying their length (longitudinal and transverse directions), or their thickness for the case of the through-thickness direction. The contact resistances were estimated to be $12 \pm 2 \mathrm{~m} \Omega$ in the longitudinal direction, $240 \pm 60 \mathrm{~m} \Omega$ in the transverse direction, and $160 \pm 20 \mathrm{~m} \Omega$ in the through-thickness direction. For the characterisation of the transverse and through-thickness electrical and thermal conductivity, two samples for each filler content were tested. To account for discrepancies from the infusion process of the GO-modified epoxy into the fibres, samples were cut near the resin inlet side and the vacuum outlet. The obtained results are quoted as the mean of the values obtained for the resin inlet and vacuum side. All samples were cut from the same plate.

\section{Thermal conductivity measurements}

The through-thickness and transverse direction thermal conductivities were measured using a steady-state technique based on a guarded hot plate (GHP) method, see Fig. 3. The advantage of steadystate techniques is that, besides the thickness of the test sample, the only additional information required to obtain the thermal conductivity is the interface resistance between the sample and the substrates $[33,34]$. The technique is based on establishing a onedimensional (1-D) heat flow through by placing the bulk of the sample between a pair of circular metallic substrates (brass plates/discs with a diameter of $50 \mathrm{~mm}$ and a thickness of $15 \mathrm{~mm}$ ) and heating using a $2 \mathrm{~W}, 12 \mathrm{~V}$ heating element attached to the base of the hot plate substrate. Steady-state conditions were defined when the temperature was exhibiting variations of less than $0.5 \mathrm{~K}$ over a period of $30 \mathrm{~min}$. To accurately obtain the heat flux, a thin film flux sensor, OMEGA HFS-4, with a sensitivity of $1.6 \mu \mathrm{V} / \mathrm{W} / \mathrm{m}^{2}$, was incorporated into the design. The DC voltage output signal from the sensor was recorded using an
Agilent technologies 34401A voltmeter. Temperature readings were conducted using K-type thermocouples connected with a Pico Technologies TC-08 thermocouple data logger. To avoid lateral heat losses, the apparatus was insulated with polymethacrylimide (PMI) foam. To mitigate the influence of surface roughness, air gaps and thermal contact resistance, a silicone-based heat transfer compound with a thermal conductivity of $3 \mathrm{~W} / \mathrm{mK}$, Electrolube HTSP was applied in the interfaces between the brass substrates and the test specimen. To assure reproducibility, an M6 bolt actuator was employed to apply a torque of 1 $\mathrm{Nm}$ a total of $0.28 \mathrm{MPa}$ of applied (average) pressure ensuring identical conditions during the measurements. This level of externally applied pressure was sufficient to ensure that surface air gaps were avoided in the interfacing material (coming in paste form).

The specimens required to measure the throughthickness thermal conductivity were circular discs of $50 \mathrm{~mm}$ in diameter, which were simply cut from the as-manufactured laminates using waterjet cutting. Manufacture of the specimens suitable for measuring the transverse thermal conductivity was rather more challenging as the heat flow has to be in the transverse direction; Fig. 4 shows how this was achieved. CFRP strips, $7 \mathrm{~mm}$ and $9 \mathrm{~mm}$ wide, were cut from the manufactured laminates and bonded together using an epoxy-based adhesive to form discs of $50 \mathrm{~mm}$ diameter such that the transverse direction of the laminate was aligned with the heat flow direction (i.e. through the thickness of the disc). Since the epoxy bond line was parallel to the heat flow direction, it was assumed that the adhesive did not affect the measurements as the total area of the bonding is about $1.5 \%$ of the total cross-sectional area of the samples.

For both the through-thickness and transverse laminate directions, samples with different thicknesses were manufactured and tested to investigate the influence of the interface conductance on the measurements. By plotting the ratio of the temperature gradient to the flux, i.e. $\Delta T / q$, versus the sample thickness $\Delta x$, the interface conductance $h$ can be estimated from the intercept with the $\Delta T / q$ axis, as indicated in Fig. $5[1,34,35]$.

Each sample was measured two or more times, and corrections to the values were made using an analogue of resistances connected in series as follows: 
Figure 3 Schematic of the testing apparatus (thermal insulation is not shown).

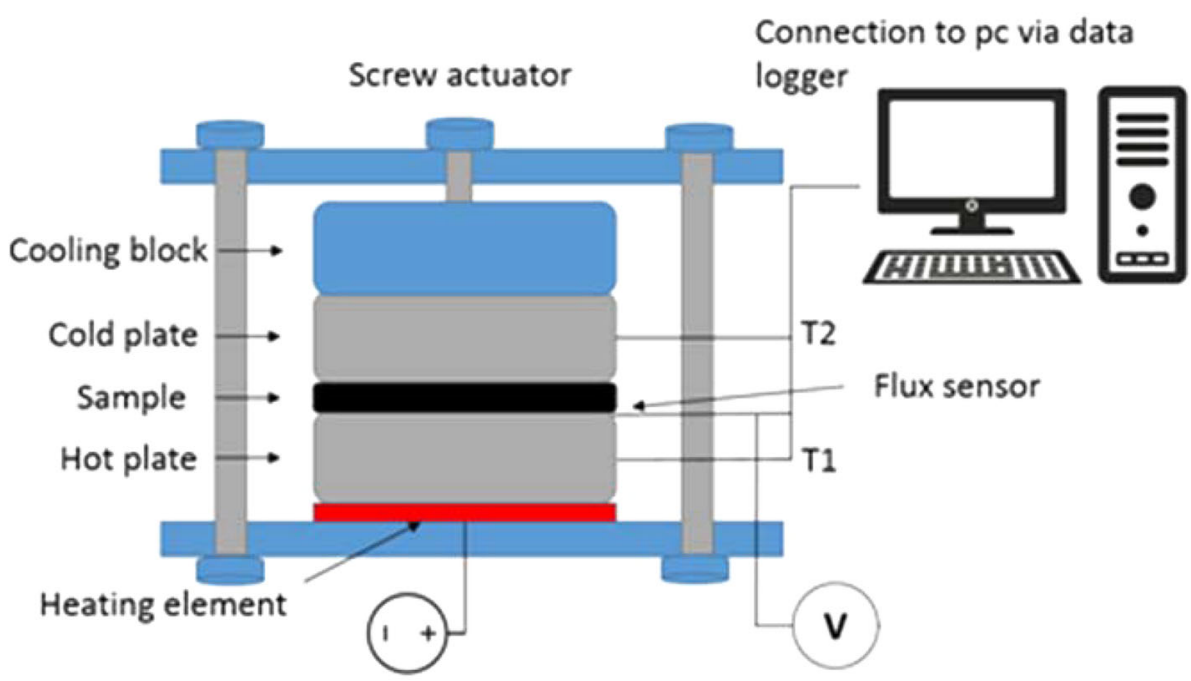

Epoxy bond line

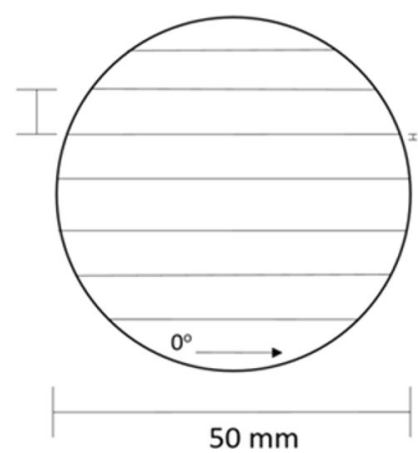

Figure 4 Schematic of the sample used for the transverse thermal conductivity measurements.

$\frac{\Delta x}{k_{\text {meas }}}=\frac{\Delta x}{k_{\text {true }}}+\frac{1}{h}$

where the ratio $\Delta x / k$ is the thermal resistance of the sample, $1 / h$ is the thermal interface resistance, $\Delta x$ is the sample thickness, $k_{\text {meas }}$ is the measured thermal conductivity, $k_{\text {true }}$ is the corrected value of thermal conductivity and $h$ is the interface conductance.

The estimations of the measurement uncertainty were realised by calculating the percentage of the deviation between the $k_{\text {true }}$ values with the thermal conductivity value obtained from the slope of the plot depicted in Fig. 5. To validate the measuring accuracy of the apparatus, measurements were carried out on sample materials with well-defined thermal conductivity values. In this study, two reference materials were used to obtain a two-point calibration: PTFE (polytetrafluoroethylene), supplied by RS components, and fused silica (quartz glass), supplied by UQG Optics, Cambridge, UK. Disc-shaped
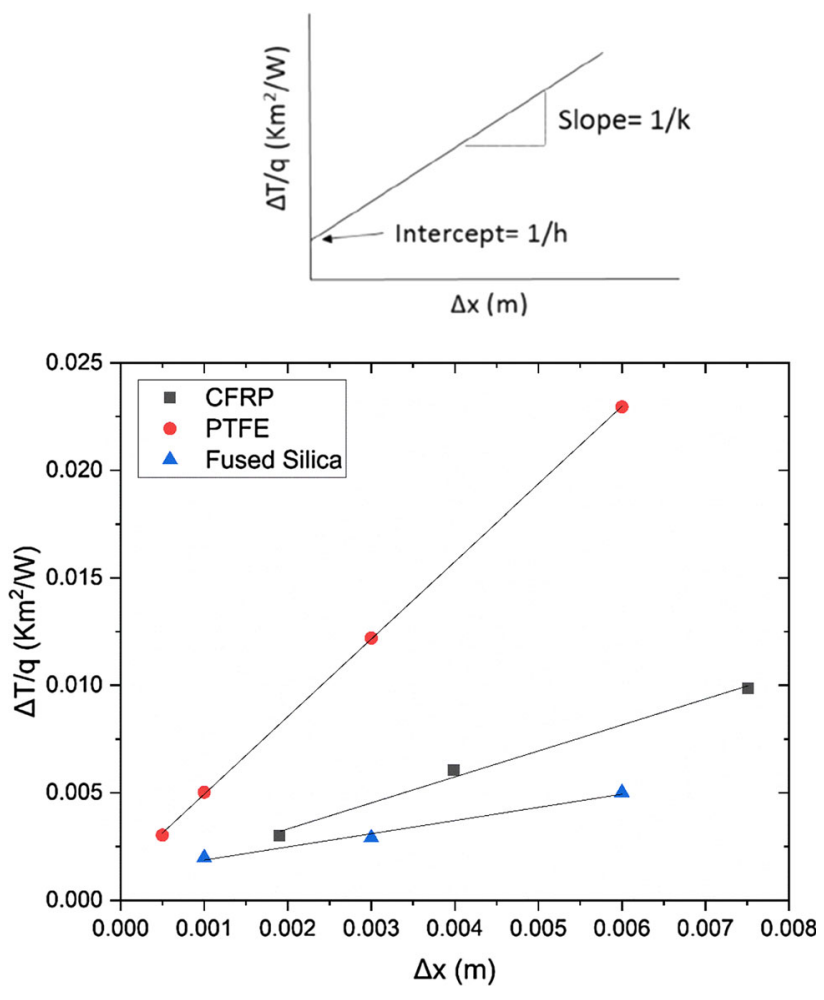

Figure 5 Ratio of temperature gradient $(\Delta T)$ to flux $(q)$ versus sample thickness $(\Delta x)$ (up) theoretical and (bottom) for the PTFE, fused silica and CFRP examined in this study.

samples with a diameter of $50 \mathrm{~mm}$ and various thicknesses were tested for both reference materials, see Fig. 5. From the results listed in Table 2, it is seen that the measured values agree well with the values stated by the suppliers and found in the literature $[34,36,37]$. The higher uncertainty observed in the measurement of the fused silica compared to the 
Table 2 Thermal conductivity values of reference materials at $20{ }^{\circ} \mathrm{C}$

\begin{tabular}{lllr}
\hline Material & Thermal conductivity $(\mathrm{W} / \mathrm{mK})$ & Thermal conductivity (measured) $(\mathrm{W} / \mathrm{mK})$ & ${\text { Interface conductance }\left(\mathrm{W} / \mathrm{m}^{2} \mathrm{~K}\right)}$ \\
\hline PTFE & $0.2-0.3[36,37]$ & $0.277 \pm 0.0015$ & 754 \\
Fused silica & $1.38[34]$ & $1.428 \pm 0.05$ & 1234 \\
\hline
\end{tabular}

Table 3 Measured electrical and thermal conductivities of reference (neat) UD CFRP laminate

\begin{tabular}{lcll}
\hline Direction & Electrical conductivity $(\mathrm{S} / \mathrm{cm})$ & \multicolumn{2}{l}{ Thermal properties } \\
\cline { 3 - 4 } & & Thermal conductivity $(\mathrm{W} / \mathrm{mK})$ & ${\text { Interface conductance }\left(\mathrm{W} / \mathrm{m}^{2} \mathrm{~K}\right)}^{\mathrm{N}}$ \\
\hline Longitudinal & $220 \pm 14$ & $\mathrm{~N} / \mathrm{A}$ & 1391 \\
Transverse & $0.561 \pm 0.001$ & $0.87 \pm 0.01$ & 1100 \\
Through thickness & $0.0543 \pm 0.0027$ & $0.77 \pm 0.02$ & \\
\hline
\end{tabular}

PTFE is associated with the GHP method. Thermal conductivity values similar to the one for fused silica are usually close to the limit of the measuring capabilities of this method [36].

\section{Interlaminar shear strength (ILSS) assessment}

To assess the impact of adding GO to the resin system, it was decided to conduct interlaminar shear strength (ILSS) testing. This test does not provide a complete overview or documentation of mechanical performance, but it does provide a good assessment of the properties of the fibre-matrix interface, thereby the stress transfer performance of the GO-modified composite system. The ILSS was evaluated using a short-beam shear test (three-point bending). The test was performed in accordance with the ASTM D2344 test standard [38]. The span to width ratio was set to $4: 1$, and the crosshead movement rate was $1 \mathrm{~mm} /$ $\mathrm{min}$. To account for manufacturing variabilities, fibre volume fraction variations and possible inhomogeneous dispersion of the filler, test specimens were cut along the resin flow direction. The mechanical tests were conducted using an Instron 5569 universal electro-mechanical testing machine with load capacity of $50 \mathrm{kN}$, and the Bluehill software from Instron was used for logging the load/displacement data. The results were presented as mean values with standard deviations based on all of the tested samples of each of the examined laminates. The ILSS was calculated using the following expression:
$\mathrm{ILSS}=0.75 *\left(\frac{P}{b * h}\right)$

where $P$ is the maximum load observed during the test, $b$ the specimen width and $h$ the specimen thickness.

\section{Results}

\section{Reference electrical and thermal conductivities}

The electrical and thermal conductivities of the reference composite material system, i.e. neat CFRP without GO-modified resin, were measured to establish a reference for comparative purposes. From the measured DC electrical conductivity results shown in Table 3, it is observed that the electrical conductivity in the through-thickness direction is almost an order of magnitude lower than in the transverse direction (i.e. in-plane and perpendicular to fibres). Furthermore, the electrical conductivity in the fibre direction is approximately three orders of magnitude greater than in the transverse direction, and four orders of magnitude higher than in the through-thickness direction. Thus, the level of anisotropy plays a significant role in determining the electrical conductivity of the reference CFRP.

The measured thermal conductivities in the transverse and through-thickness directions are also given in Table 3. It is seen that the measured values are indicative of the anisotropic behaviour, with the transverse direction thermal conductivity being 
about $13 \%$ higher than the value in the throughthickness direction.

\section{Electrical conductivity}

The influence of GO filler content on the electrical conductivity in the transverse direction is shown in Fig. 6. It is seen that the addition of GO leads to a slight increase in the conductivity values. No substantial increase was observed for low filler contents, i.e. below $1.26 \mathrm{vol} \%$, but the effect becomes more pronounced at filler contents above $2.52 \mathrm{vol} \%$ where

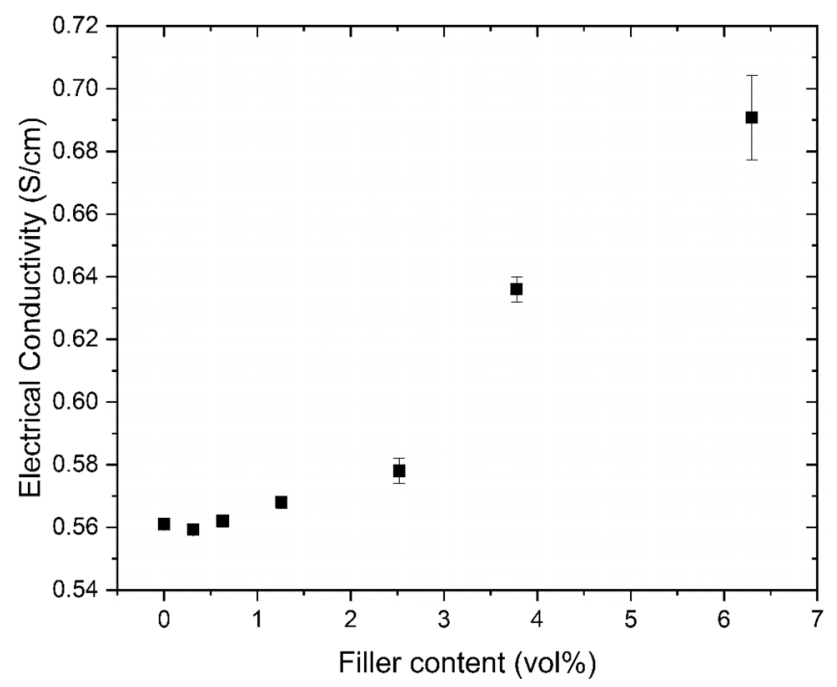

Figure 6 Transverse electrical conductivity as a function of GO vol\% dispersed into the polymer matrix.

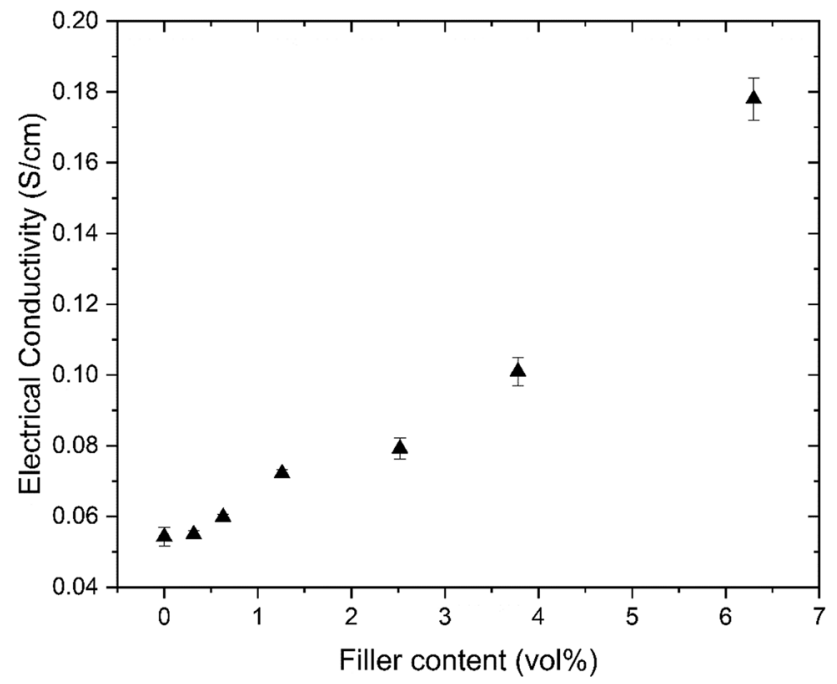

Figure 7 Through-thickness electrical conductivity as a function of GO dispersed into the polymer matrix. a nonlinear increase occurs with further addition of GO reaching its maximum value of approx. $0.7 \mathrm{~S} / \mathrm{cm}$ at $6.3 \mathrm{vol} \%$.

Figure 7 shows the measured electrical conductivity in the through-thickness direction as a function of the GO filler content. A similar trend as in the transverse direction is observed. At low filler contents, i.e. $0.32 \mathrm{vol} \%$ and $0.63 \mathrm{vol} \%$, there appears to be no effect of the GO filler. The effects GO filler becomes more prominent from contents of $1.26 \mathrm{vol} \%$ and above, where a steady increase is observed. Finally, a threefold increase in the electrical conductivity compared to the neat system was achieved with $6.3 \mathrm{vol} \%$ GO filler providing an approximate value of $0.18 \mathrm{~S} / \mathrm{cm}$.

\section{Thermal conductivity}

Figure 8 shows that different GO filler contents do not have any significant influence on the measured transverse thermal conductivity with only minor changes from the neat CFRP system value (see Table 3).

The through-thickness thermal conductivity is presented in Fig. 9 as a function of the GO filler. When filler contents below $2.52 \mathrm{vol} \%$ are used, the thermal conductivity is not significantly affected by the addition of GO. Above $2.52 \mathrm{vol} \%$, the thermal conductivity increases approximately linearly up to the highest GO filler content, i.e. 6.3 vol\%.

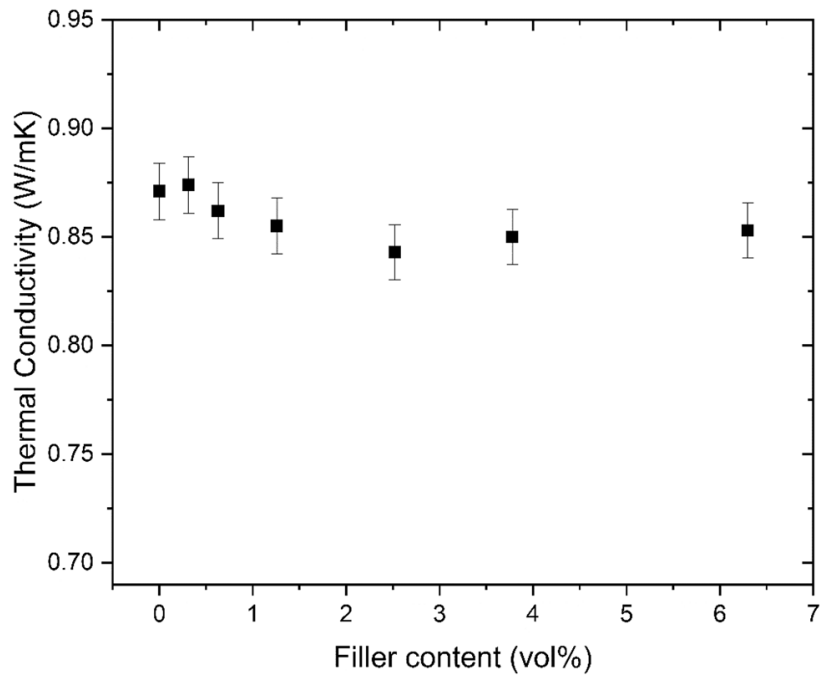

Figure 8 Transverse thermal conductivity results as a function of the GO filler content. 


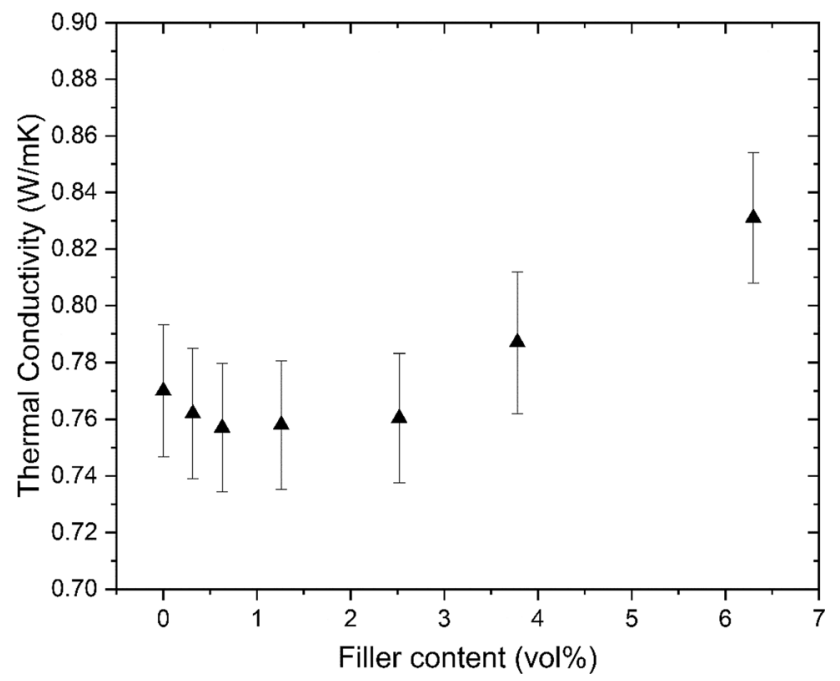

Figure 9 Through-thickness thermal conductivity as a function of the GO filler content.

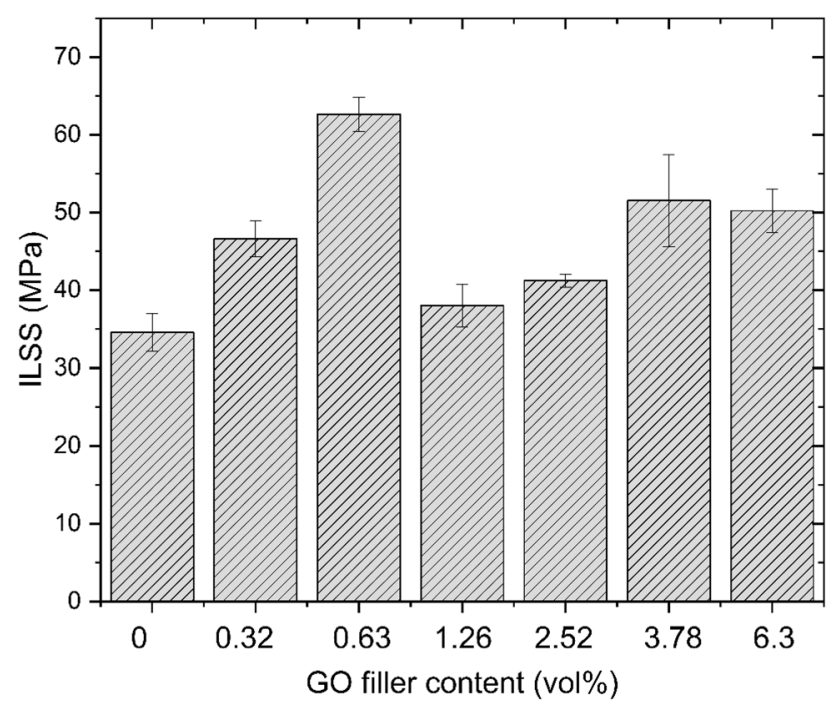

Figure 10 Interlaminar shear strength as function of GO filler content.

\section{Interlaminar shear strength (ILSS)}

The measured ILSS results are shown in Fig. 10. It is seen that the addition of relatively low GO contents, i.e. $0.32 \mathrm{vol} \%$ and $0.63 \mathrm{vol} \%$, provides significant improvements in the ILSS relative to the CFRP neat system, with increases of $29.7 \%$ and $58.4 \%$, respectively. For higher GO filler contents, i.e. 1.26 and 2.52 vol\%, there was no significant effect on the measured ILSS relative to the neat CFRP, although modest improvements in the ILSS of $9.5 \%$ and $17.6 \%$ are seen. The CFRPs with 3.78 vol\% and 6.3 vol\% GO produced an ILSS that is $37 \%$ higher than the neat CFRP system.

\section{Discussion}

\section{Morphology and dispersion of GO filler}

From the SEM images, see Fig. 11, it is seen that the morphology of the inclusions dispersed into the CFRP laminate appears to be similar to the dimensions stated by the supplier, see Fig. 11b. As expected, inclusions in the form of aggregates are observed in high filler contents, but they cannot be considered as a case of severe aggregation. Aggregates with dimensions similar to the fibre diameter will significantly affect both the infusion process and the mechanical response of the laminate. This is because of the oxygen groups that exist in GO that contribute to larger intramolecular distances, thus creating weaker bonds, making it easier for exfoliation from thicker stacks into small platelets to occur, even using simpler methods such as planetary mixing [29, 31]. Furthermore, the GO particles are seen to be located in the close vicinity of the fibre surfaces, which is indicative of good compatibility of the filler with the epoxy matrix and the epoxy-based sizing of the fibres. In the case of high GO filler loading, i.e. 6.3 $\mathrm{vol} \%$, it is worth noting the concentration of filler adjacent to the fibres, Fig. 11d, where GO appears to be attached to both the epoxy and the fibres forming an interfacial region. This particular feature suggests compatibility between GO and epoxy resin and can be expected to provide improvements in the electrical and thermal conductivities as well as the mechanical stress transfer capability.

\section{Electrical conductivity}

From the characterisation of the electrical conductivity of the neat CFRP system, see Table 3, it is evident that the conductivity in the transverse and through-thickness directions is very poor compared with the longitudinal direction, which is dominated by the fibre properties. An initial assumption would be that the electrical conductivity in the transverse and through-thickness directions of a UD CFRP laminate should be identical or very similar. However, as evidenced by Table 3, the through-thickness conductivity is about one order of magnitude lower 
Figure 11 Scanning electron microscopy images for the samples containing: a neat CFRP, b 0.63 vol\% GO (fibre surface view), c 6.3 vol\% GO (interlaminar region) and $\mathbf{d} 6.3$ vol\% (fibre surface view).
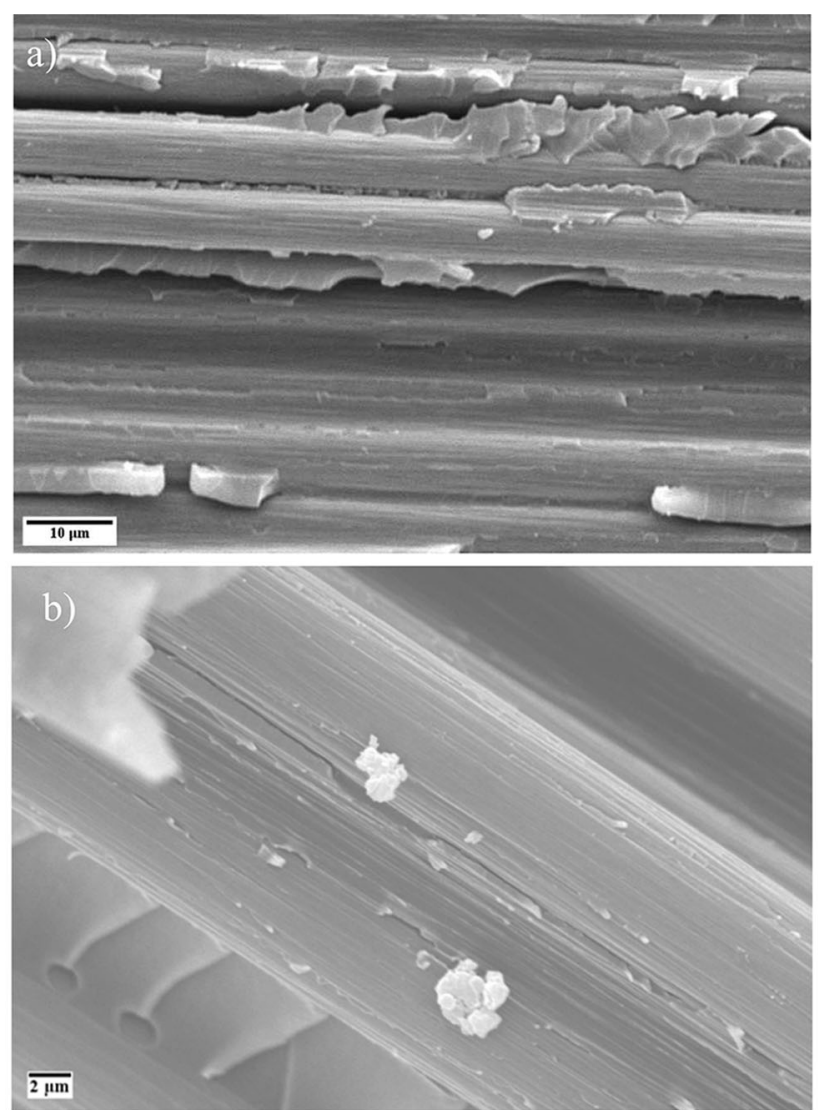

GO in contact

with the fibres
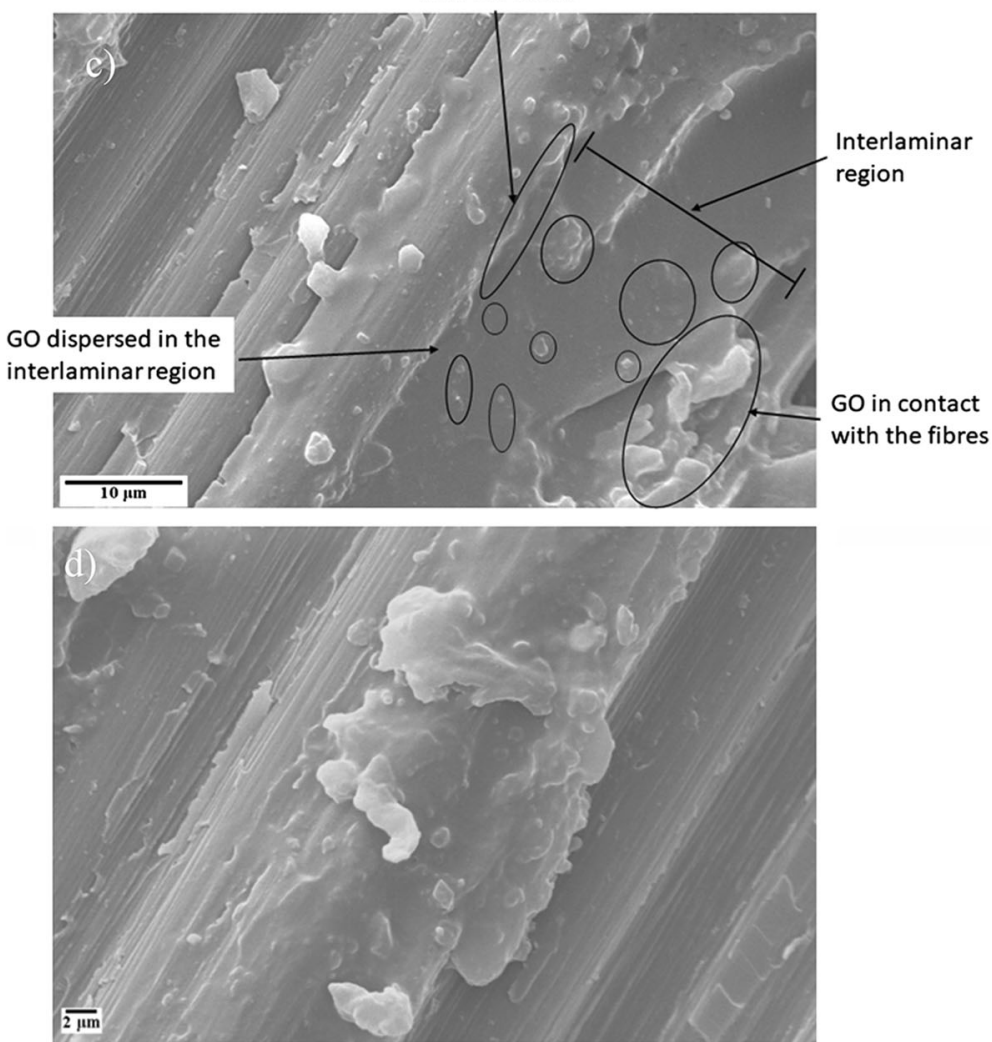
than in the transverse direction. The reason for this is that in both vacuum-infused and prepreg-based laminates, resin-rich interlaminar regions are formed leading to a significant reduction in the electrical conductivity in the through-thickness direction. This effect has also been reported in previous research $[4,8]$.

For the transverse electrical conductivity, the measurements (see Fig. 6) show that the low contents of GO up to $2.52 \mathrm{vol} \%$ do not have a significant effect, exhibiting values that are very similar to the neat CFRP properties. However, at high filler contents, 6.3 $\mathrm{vol} \%$, an increase in the electrical conductivity is observed. Although this increase cannot be described as substantial, it is not expected that drastic increases in the transverse direction will occur since current conduction is dominated by fibre-to-fibre contact points [39]. Considering that the laminates manufactured in this study have fibre volume fractions well above the percolation threshold, which is approx. $40 \mathrm{vol} \%$ [40], the addition of high contents of GO can only decrease the contact resistance between the fibres or interconnect adjacent fibres at multiple points along the bulk of the laminate. Thus, by reducing the intra-lamina resistance, the composite behaves similarly to a laminate with higher fibre volume fraction. Hence, the observed nonlinear increase for high GO contents, in this case above 2.52 vol\%. The above mechanism is supported by the SEM characterisation (see Fig. 11c), where GO flakes appear to interconnect adjacent carbon fibres, within the same lamina.

Considering the through-thickness direction measurements (see Fig. 7), it is seen that the GO filler content does have significant influence on the electrical conductivity, and a nonlinear increase can be observed with the addition of GO. At low GO contents, i.e. $0.32 \mathrm{vol} \%$ and $0.63 \mathrm{vol} \%$, a modest increase in the conductivity is seen. Further increasing the GO content, i.e. to $1.26,2.52$ and 3.78 vol\%, leads to a more substantial rise of the conductivity as the higher volume of conducting inclusions dispersed into the interlaminar region polymer facilitate the current flow through the laminate bulk. Since interlaminar regions are dominated by the polymer matrix, the associated conduction mechanism, in this case tunnelling through the dispersed GO flakes, can be treated as in a polymer composite, where the dependency of the electrical conductivity from the filler content is nonlinear [41]. For the highest GO filler content, i.e. $6.3 \mathrm{vol} \%$, a threefold increase in the electrical conductivity is observed, where the conductivity reaches the same order of magnitude as in the transverse direction, i.e. $0.18 \mathrm{~S} / \mathrm{cm}$ and $0.56 \mathrm{~S} / \mathrm{cm}$, respectively. The explanation for this behaviour is supported by the SEM images, see Fig. 11c, which indicate that GO inclusions interconnect the adjacent laminae by forming conducting paths through the interlaminar region. In the literature, it has been reported that different fillers and filler incorporation methods can also provide improvement for comparable material and processing systems suitable for large volume manufacturing. For instance, the use of GNPs in the fibre's sizing has shown improvements with values up to $0.07 \mathrm{~S} / \mathrm{cm}$ [10], which is less than the improvement reported here. The incorporation of several types of CNTs has shown its potential with through-thickness values ranging from $\sim 10^{-3} \mathrm{~S} / \mathrm{cm}$ [8, 42] up to $0.089 \mathrm{~S} / \mathrm{cm}$ [43], which is similar to that reported here for the lower filler contents. However, it should be noted that it is difficult to increase the content of CNTs due to the increase in viscosity of the polymer matrix and possible degradation of some of the mechanical properties. Although the increase observed in this study is significant, it is clear that to produce a through-thickness conductivity identical to the conductivity in the transverse direction, significantly higher quantities of filler need to be infused into the laminate to deliver high degree of percolation into the matrix material. Ideally, this would provide further increase in the conductivity since the physical current-conducting paths are formed prior to the infusion. However, higher levels of filler loading will make the matrix material difficult to handle as the viscosity increases drastically when percolation is observed and it will prevent a good fibres consolidation to the resin during the infusion process. Also, higher GO filler contents are likely to have a degrading effect on the ILSS.

\section{Thermal conductivity}

Similar trends as for the electrical conductivities were observed for the thermal conductivities in the transverse and through-thickness directions, see Table 3 and Figs. 8 and 9. Epoxy-rich interlaminar regions decrease the through-thickness thermal conductivity relative to the transverse thermal conductivity by approximately $12 \%$, which can be interpreted as the rate of improvement that needs to be achieved to 
obtain the same thermal conductivities in the through-thickness and transverse directions. As indicated by the measurements in the throughthickness direction, significant thermal conductivity improvements are only observed when high amounts of conducting filler are added to the epoxy matrix, i.e. $6.3 \mathrm{vol} \%$ GO. The associated mechanism is the enhancement of thermal conduction along the platelet-shaped GO inclusions that occur in the interlaminar region of the thermally insulating polymer matrix. Unlike the electrical conductivity, improvements in thermal conductivity of a polymer require significantly higher filler loadings, which is not surprising since the values of thermal conductivities for GO and epoxy are only two orders of magnitude apart [18]. Hence, improved thermal conductivities can only be seen for 3.78 and $6.3 \mathrm{vol} \%$. Although the addition of a thermally conducting filler can assist the thermal conduction process, particular fillers and manufacturing/dispersion processes can further affect the rate of improvement, for example by aligning GO inclusions in a particular direction which is consistent with the conventional effective medium field theory [44]. In the case of GO filler, the oxygen content represents impurities in the crystalline structure of the filler and can greatly affect the thermal conductivity of the filler itself $[22,23]$. For the as-received GO used in this study, the oxygen content was between 5 and 10\%, which can be considered as low, and, thus, the thermal conductivity of the filler will not be affected drastically. Molecular dynamics has shown that the thermal conductivity of GO can be reduced down to $8.8 \mathrm{~W} / \mathrm{mK}$ when the oxygen coverage is close to $20 \%$ [23]. Similar results were reported after characterising the thermal conductivity of free-standing GO films [22]. For low filler contents, up to $2.52 \mathrm{vol} \%$, the added filler is not sufficient to provide additional conduction in the interlaminar region, and the measured thermal conductivities are of the same magnitude as for the neat CFRP laminate. However, for 6.3 vol\% filler content, the throughthickness thermal conductivity increased up to a point of reaching almost identical values as observed in the transverse direction of the neat CFRP. This is probably related to a non-uniform distribution of GO flakes, as at high GO vol\% the flakes mainly accumulate in the interlaminar region making the local vol\% of GO higher than the average in the laminate's bulk. The highest thermal conductivity provided in the present study is $0.83 \mathrm{~W} / \mathrm{mK}$; it is the case that higher values have been reported in the literature [35]. However, many other factors have an effect on the thermal conductivity of nano-reinforced laminates such as the carbon fibre type, the fibre volume fraction, the thermal conductivity of the epoxy, the manufacturing method, the method of adding the nano-filler into the composite and the measurement temperature. Here, the important factor is the suitability of the materials and the process for large volume manufacturing (as discussed in the previous section of the paper). Leaving that aside, similar values, $0.86 \mathrm{~W} / \mathrm{mK}$, have been reported for laminates containing GNPs, for the same filler content [16], but also for a significantly lower filler content of $0.5 \mathrm{wt} \%$, $0.84 \mathrm{~W} / \mathrm{mK}$ [45], but the GNPs were deposited directly onto the carbon fabric, adding both to cost and the complexity of the manufacturing process. Significantly lower values, approx. $0.53 \mathrm{~W} / \mathrm{mK}$, were observed in laminates containing both GNPs and SNPs, however, at a much lower fibre volume fraction [7].

The addition of GO makes little difference to the transverse direction thermal conductivity (Fig. 8). Unlike the through-thickness direction, in the transverse direction, there are no interlaminar resin-rich regions, which means that the heat flows mainly through the fibres passing small epoxy gaps between them. The main thermal resistance mechanism in this case would be heat flux funnelling towards fibre contacts. Hence, the addition of GO filler cannot provide the noticeable improvements observed for the through-thickness direction. The marginal decrease with increasing GO filler content, although within the experimental uncertainty, can be attributed to fibre contact topology changes caused by fibre volume fraction variations during the infusion process.

\section{ILSS}

From the ILSS tests conducted for varying GO filler contents, see Fig. 10, it is observed that the addition of GO into the epoxy matrix leads to increased ILSS values, i.e. to improved matrix-fibre stress transfer. For low GO filler contents, i.e. 0.32 vol $\%$ and 0.63 vol\%, a sharp increase in the ILSS is seen. The observed improvement can be attributed to the planar geometry of the GO filler agglomerates (flakes), with large surface areas, leading to enhanced filler/matrix adhesion [46, 47]. At low filler contents, 
it can be assumed that the filler is homogenously dispersed in the bulk of the laminate, which enables the GO flakes to effectively act as crack deflectors, thus suppressing further crack propagation into the epoxy matrix. In addition, as observed in the SEM images (Fig. 11), the rough or wrinkled GO flake surfaces contribute to the interlocking of the polymer matrix onto the GO. For intermediate filler contents, i.e. $1.26 \mathrm{vol} \%$ and $2.52 \mathrm{vol} \%$, some aggregation is observed, and this can cause the formation of local stress concentration zones leading to decreased ILSS values compared to the samples containing finely dispersed GO flakes, while still providing improved behaviour in comparison to the neat CFRP. By further increasing the GO filler content, i.e. $6.3 \mathrm{vol} \%$, notwithstanding the aggregation of the fillers, the higher dispersed volume of GO flakes provides a secondary reinforcement and strengthens both the epoxy/carbon fibre interphase and polymer interlaminar regions [48]. This explains the substantially increased ILSS value relative to that in the intermediate GO filler content samples and is supported by the literature where improvements in ILSS are reported for other means of filling, including the incorporation of GO into the sizing and depositing GNPs directly onto the carbon fabric. Similar results to those found in the present paper are reported $[5,45]$, thus confirming the efficiency of incorporating GO filler directly in the bulk matrix.

\section{Conclusions}

It was demonstrated that GO-reinforced CFRP laminates manufactured using VARTM and bulk matrix nano-filler modification exhibit improved electrical and thermal through-thickness properties, as well as improved ILSS values. Five different GO filler loads were investigated: $0.32,0.63,2.52,3.78$ and $6.3 \mathrm{vol} \%$. The electrical conductivities have been shown to be far more sensitive to the addition of GO than the thermal conductivities, with the highest levels of improvement observed in the through-thickness direction, whilst the transverse direction electrical conductivity was found not to be significantly affected. The observations regarding ILSS results suggest that interphase tailoring can be achieved by bulk matrix modification with the GO filler addition. This further suggests that it is feasible to manufacture CFRP laminates that can be utilised in applications

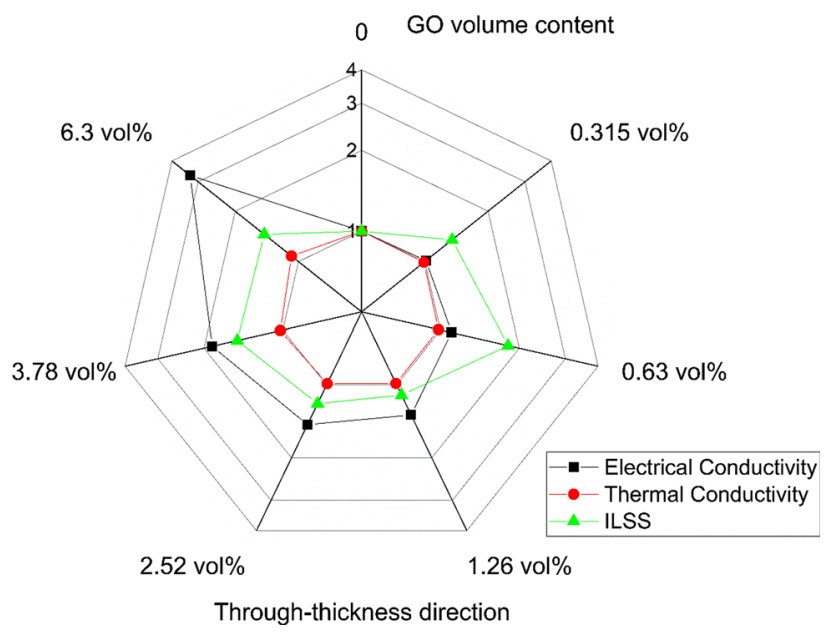

Figure 12 Comparative plot of the relative improvement in the through-thickness electrical and thermal conductivities as well as ILSS as a function of the GO filler content. All values quoted are normalised with regard to the neat CFRP laminate properties.

where electric current or heat is expected to be introduced into the CFRP structure, whilst at the same time retaining or improving the ILSS, which is typically a very important parameter of mechanical design (albeit not the only important design parameter). The improved electrical (in particular) and thermal conductivities have the potential to improve the overall material performance by acting as a means of mitigation of electrically or thermally induced damage locally. An overview summary of the improvements observed for the through-thickness electrical and thermal conductivities, as well as the ILSS, relative to the properties of neat CFRP UD laminates is shown in Fig. 12, which provides an overview and road map to the multifunctional behaviour and potential improvements in GO-reinforced UD CFRP material systems for structural applications.

\section{Acknowledgements}

The research was sponsored by the Marie Sklodowska Curie Actions, Innovative Training Networks (ITN), Call: H2020-MSCA-ITN-2014, as part of the 642771 SPARCARB project. The authors are grateful to Mr. Orestis Vryonis, University of Southampton, for his assistance during the dispersion of GO filler into epoxy resin, and Mrs Allison Shaw, also University of Southampton, for the SEM microscopy. The mechanical testing described in the 
paper was conducted in the Testing and Structures Research Laboratory (TSRL) at the University of Southampton, and the authors are also grateful for the support received from Dr Andy Robinson, the TSRL Principal Experimental Officer. The authors thank Garmor Inc. (http://www.garmortech.com/) for providing the GO used in this study.

Open Access This article is distributed under the terms of the Creative Commons Attribution 4.0 International License (http://creativecommons.org/ licenses/by/4.0/), which permits unrestricted use, distribution, and reproduction in any medium, provided you give appropriate credit to the original author(s) and the source, provide a link to the Creative Commons license, and indicate if changes were made.

\section{References}

[1] Han S, Lin JT, Yamada Y, Chung DDL (2008) Enhancing the thermal conductivity and compressive modulus of carbon fiber polymer-matrix composites in the through-thickness direction by nanostructuring the interlaminar interface with carbon black. Carbon N Y 46:1060-1071

[2] Yao X, Falzon BG, Hawkins SC, Tsantzalis S (2018) Aligned carbon nanotube webs embedded in a composite laminate: A route towards a highly tunable electro-thermal system. Carbon N Y 129:486-494. https://doi.org/10.1016/ j.carbon.2017.12.045

[3] Yao X, Hawkins SC, Falzon BG (2018) An advanced antiicing/de-icing system utilizing highly aligned carbon nanotube webs. Carbon N Y 136:130-138. https://doi.org/10. 1016/j.carbon.2018.04.039

[4] Pozegic TR, Anguita JV, Hamerton I et al (2016) Multifunctional carbon fibre composites using carbon nanotubes as an alternative to polymer sizing. Sci Rep 6:37334

[5] Zhang X, Fan X, Yan C et al (2012) Interfacial microstructure and properties of carbon fiber composites modified with graphene oxide. ACS Appl Mater Interfaces 4:1543-1552

[6] Yao X, Gao X, Jiang J et al (2018) Comparison of carbon nanotubes and graphene oxide coated carbon fiber for improving the interfacial properties of carbon fiber/epoxy composites. Compos Part B Eng 132:170-177

[7] Kandare E, Khatibi AA, Yoo S et al (2015) Improving the through-thickness thermal and electrical conductivity of carbon fibre/epoxy laminates by exploiting synergy between graphene and silver nano-inclusions. Compos Part A Appl Sci Manuf 69:72-82. https://doi.org/10.1016/j.compositesa. 2014.10.024
[8] El Sawi I, Olivier PA, Demont P, Bougherara H (2012) Processing and electrical characterization of a unidirectional CFRP composite filled with double walled carbon nanotubes. Compos Sci Technol 73:19-26. https://doi.org/10. 1016/j.compscitech.2012.08.016

[9] Adak NC, Chhetri S, Murmu NC et al (2019) Experimental and numerical investigation on the mechanical characteristics of polyethylenimine functionalized graphene oxide incorporated woven carbon fibre/epoxy composites. Compos Part B Eng 156:240-251. https://doi.org/10.1016/j.compo sitesb.2018.08.086

[10] Qin W, Vautard F, Drzal LT, Yu J (2015) Mechanical and electrical properties of carbon fiber composites with incorporation of graphene nanoplatelets at the fiber-matrix interphase. Compos Part B Eng 69:335-341. https://doi.org/ 10.1016/j.compositesb.2014.10.014

[11] Mahmood H, Vanzetti L, Bersani M, Pegoretti A (2018) Mechanical properties and strain monitoring of glass-epoxy composites with graphene-coated fibers. Compos Part A Appl Sci Manuf 107:112-123. https://doi.org/10.1016/j.co mpositesa.2017.12.023

[12] Wang F, Cai X (2018) Improvement of mechanical properties and thermal conductivity of carbon fiber laminated composites through depositing graphene nanoplatelets on fibers. J Mater Sci. https://doi.org/10.1007/s10853-018-309 7-3

[13] Yamamoto N, Guzman de Villoria R, Wardle BL (2012) Electrical and thermal property enhancement of fiber-reinforced polymer laminate composites through controlled implementation of multi-walled carbon nanotubes. Compos Sci Technol 72:2009-2015. https://doi.org/10.1016/j.compsc itech.2012.09.006

[14] Gaztelumendi I, Chapartegui M, Seddon R et al (2017) Enhancement of electrical conductivity of composite structures by integration of carbon nanotubes via bulk resin and/ or buckypaper films. Compos Part B Eng 122:31-40

[15] Domingues D, Logakis E, Skordos AAA (2012) The use of an electric field in the preparation of glass fibre/epoxy composites containing carbon nanotubes. Carbon N Y 50:2493-2503. https://doi.org/10.1016/j.carbon.2012.01.072

[16] Kostagiannakopoulou C, Fiamegkou E, Sotiriadis G, Kostopoulos V (2016) Thermal conductivity of carbon nanoreinforced epoxy composites. J Nanomater 2016:1-12. h ttps://doi.org/10.1155/2016/1847325

[17] Wu S, Ladani RB, Ravindran AR et al (2017) Aligning carbon nanofibres in glass-fibre/epoxy composites to improve interlaminar toughness and crack-detection capability. Compos Sci Technol 152:46-56. https://doi.org/10.1 016/j.compscitech.2017.09.007 
[18] Burger N, Laachachi A, Ferriol M et al (2016) Review of thermal conductivity in composites: mechanisms, parameters and theory. Prog Polym Sci 61:1-28

[19] As-Y Lee, Chong M-H, Park M et al (2014) Effect of chemically reduced graphene oxide on epoxy nanocomposites for flexural behaviors. Carbon Lett 15:67-70

[20] Stankovich S, Dikin DA, Dommett GHB et al (2006) Graphene-based composite materials. Nature 442:282

[21] Atif R, Shyha I, Inam F (2016) Mechanical, thermal, and electrical properties of graphene-epoxy nanocomposites-a review. Polymers (Basel) 8:281

[22] Renteria JD, Ramirez S, Malekpour H et al (2015) Strongly anisotropic thermal conductivity of free-standing reduced graphene oxide films annealed at high temperature. Adv Funct Mater 25:4664-4672

[23] Mu X, Wu X, Zhang T et al (2014) Thermal transport in graphene oxide-from ballistic extreme to amorphous limit. Sci Rep 4:3909

[24] Hummers WS Jr, Offeman RE (1958) Preparation of graphitic oxide. J Am Chem Soc 80:1339

[25] Romero D, Montanyà J, Vinck J (2014) Test and simulation of lightning current distribution on a wind turbine blade. In: 2014 International Conference on lightning protection (ICLP), IEEE, pp 1720-1724

[26] Wang Y, Hu W (2017) Investigation of the effects of receptors on the lightning strike protection of wind turbine blades. IEEE Trans Electromagn Compat 59:1180-1187

[27] Mishnaevsky L, Branner K, Petersen HN et al (2017) Materials for wind turbine blades: an overview. Materials (Basel) 10:1285

[28] Ma P-C, Zhang Y (2014) Perspectives of carbon nanotubes/ polymer nanocomposites for wind blade materials. Renew Sustain Energy Rev 30:651-660

[29] Vryonis O, Harrell TM, Andritsch T, Vaughan AS, Lewin PL et al (2018) Solvent mixing and its effect on epoxy resin filled with graphene oxide. In: 2018 IEEE 2nd international conference on dielectrics (ICD). Budapest, Hungary, pp 1-4

[30] Chen H, Jacobs O, Wu W et al (2007) Effect of dispersion method on tribological properties of carbon nanotube reinforced epoxy resin composites. Polym Test 26:351-360

[31] Wu S, Ladani RB, Zhang J et al (2015) Aligning multilayer graphene flakes with an external electric field to improve multifunctional properties of epoxy nanocomposites. Carbon N Y 94:607-618

[32] Krieg AS, King JA, Jaszczak DC et al (2018) Tensile and conductivity properties of epoxy composites containing carbon black and graphene nanoplatelets. J Compos Mater 52:3909-3918. https://doi.org/10.1177/0021998318771460

[33] Rolfes R, Hammerschmidt U (1995) Transverse thermal conductivity of CFRP laminates: a numerical and experimental validation of approximation formulae. Compos Sci Technol 54:45-54

[34] Tan JC, Tsipas SA, Golosnoy IO et al (2006) A steady-state $\mathrm{Bi}$-substrate technique for measurement of the thermal conductivity of ceramic coatings. Surf Coat Technol 201:1414-1420

[35] Han S, Chung DDL (2011) Increasing the through-thickness thermal conductivity of carbon fiber polymer-matrix composite by curing pressure increase and filler incorporation. Compos Sci Technol 71:1944-1952

[36] Chen H, Ginzburg VV, Yang J et al (2016) Thermal conductivity of polymer-based composites: fundamentals and applications. Prog Polym Sci 59:41-85

[37] Price DM, Jarratt M (2002) Thermal conductivity of PTFE and PTFE composites. Thermochim Acta 392-393:231-236

[38] ASTM D2344/D2344M-16 (2016) STM for S-BS of PMCM and TL ASTM D2344/D2344M-16, standard test method for short-beam strength of polymer matrix composite materials and their laminates. ASTM International, West Conshohocken, PA. www.astm.org. Accessed 20 Nov 2018

[39] Schulte KK, Baron C (1989) Load and failure analyses of CFRP laminates by means of electrical resistivity measurements. Compos Sci Technol 36:63-76. https://doi.org/10.10 16/0266-3538(89)90016-X

[40] Park JB, Hwang TK, Kim HG, Doh YD (2007) Experimental and numerical study of the electrical anisotropy in unidirectional carbon-fiber-reinforced polymer composites. Smart Mater Struct 16:57-66. https://doi.org/10.1088/09641726/16/1/006

[41] Gu J, Liang C, Zhao X et al (2017) Highly thermally conductive flame-retardant epoxy nanocomposites with reduced ignitability and excellent electrical conductivities. Compos Sci Technol 139:83-89. https://doi.org/10.1016/j.compscite ch.2016.12.015

[42] Lin Y, Gigliotti M, Lafarie-Frenot MC et al (2015) Experimental study to assess the effect of carbon nanotube addition on the through-thickness electrical conductivity of CFRP laminates for aircraft applications. Compos Part B Eng 76:31-37. https://doi.org/10.1016/J.COMPOSITESB.2015.0 2.015

[43] Bekyarova E, Thostenson ET, Yu A et al (2007) Multiscale carbon nanotube-carbon fiber reinforcement for advanced epoxy composites. Langmuir 23:3970-3974

[44] Hiroshi H, Minoru T (1986) Equivalent inclusion method for steady state heat conduction in composites. Int J Eng Sci 24:1159-1172

[45] Wang F, Cai X (2019) Improvement of mechanical properties and thermal conductivity of carbon fiber laminated composites through depositing graphene nanoplatelets on 
fibers. J Mater Sci 54:3847-3862. https://doi.org/10.1007/ s10853-018-3097-3

[46] Rafiee MA, Rafiee J, Wang Z et al (2009) Enhanced mechanical properties of nanocomposites at low graphene content. ACS Nano 3:3884-3890

[47] Surnova A, Balkaev D, Musin D et al (2019) Fully exfoliated graphene oxide accelerates epoxy resin curing, and results in dramatic improvement of the polymer mechanical properties. Compos Part B Eng 162:685-691. https://doi.org/10.1016/J. COMPOSITESB.2019.01.020
[48] Karger-Kocsis J, Mahmood H, Pegoretti A (2015) Recent advances in fiber/matrix interphase engineering for polymer composites. Prog Mater Sci 73:1-43. https://doi.org/10.101 6/j.pmatsci.2015.02.003

Publisher's Note Springer Nature remains neutral with regard to jurisdictional claims in published maps and institutional affiliations. 